\title{
MODELLING WIDE-ANGLE LENS CAMERAS FOR METROLOGY AND MAPPING APPLICATIONS
}

\author{
D. Jarron ${ }^{1} *$, M. Shahbazi ${ }^{1}$, D. Lichti ${ }^{1}$, R. Radovanovic ${ }^{2}$ \\ ${ }^{1}$ Dept. of Geomatics Engineering, University of Calgary, T2N 1N4 Calgary, Alberta, Canada \\ - (dmjarron, mozhdeh.shahbazi, ddlichti)@ucalgary.ca \\ ${ }^{2}$ McElhanney Geomatics Engineering Ltd., T2G 0Y4 Calgary, AB, Canada - rradovanovic@mcelhanney.net
}

\section{ICWG II/III: Pattern Analysis in Remote Sensing}

KEY WORDS: Ladybug Camera, Fisheye Camera Calibration, GoPro

\begin{abstract}
:
Wide-angle lenses typically offer fields of view greater than $70^{\circ}$, which are utilized in a variety of imaging, mapping, and navigation applications. Wide-angle lenses are commonly modelled using the central perspective model, compensating for lens distortions through a series of additional parameters. The more extreme the distortions, the further the reality of the lens matches the collinearity equations that define the central perspective model. Fisheye lenses are modelled differently because their fields of view are so wide (typically $180^{\circ}$ ) that the collinearity model is not applicable. This work studied the effects of modelling wide-angle lenses using both the conventional central perspective model and the fisheye model to determine which model best fits the observations and models the distortions more precisely and accurately. These results were produced by generating observations in a dedicated indoor calibration facility at the University of Calgary: an $11 \mathrm{~m}$ x $11 \mathrm{~m}$ x $4 \mathrm{~m}$ field comprising 291 signalized photogrammetry targets. Multiple freenetwork, self-calibrating bundle adjustments were performed using different models and different cameras. The results of the selfcalibrating bundle adjustments were then utilized in a check adjustment on independent sets of check images to validate their accuracy. Two cameras, a Ladybug5 and a GoPro Hero5, were tested. The GoPro was also calibrated using a checkerboard target pattern, and the results were compared to those of the $3 \mathrm{D}$ calibration target-field. The results of the bundle adjustments determined that the fisheye model describes the distortions more precisely in both wide-angle camera systems.
\end{abstract}

\section{INTRODUCTION}

\subsection{Contributions}

Wide-angle lenses tend to have short focal lengths and wide fields of view (FOV), usually greater than $70^{\circ}$. Cameras constructed with wide-angle lenses are useful for a variety of applications, which recently have begun to include metrology and surveying applications, often as part of a mobile mapping system (Puente et al., 2012; Cui et al., 2016). The large field of view of this type of camera is useful for mobile mapping applications, as it can allow for fewer images to be taken to provide full coverage of the area. Some camera systems, such as the Ladybug5, generate a panoramic field of view by stitching together the imagery captured from multiple overlapping wideangle cameras. Various models of GoPro cameras also have begun to be utilized in mobile mapping applications, especially on unmanned aerial vehicles (UAVs) where their lightweight construction is an useful attribute (Possoch et al., 2016). Wide and super-wide lens cameras are also utilized in indoor mapping and navigation, where having near- $360^{\circ}$ visibility in confined spaces is necessary(Boulianne et al., 1997; da Silva et al., 2017). As wide-angle lens cameras become more common in mapping applications, where precision and accuracy are essential, it is critical to calibrate these cameras as accurately as possible. Radial distortions on wide-angle lenses tend to be very large, and many of their output images resemble full-frame images of fisheye cameras. This paper investigates the similarities between wide-angle lens cameras and fish-eye cameras and tests a variety of modelling approaches on two wide-angle lens camera systems. This paper utilizes multiple fisheye lens models and central perspective models to determine the most accurate way to model and calibrate these types of cameras, using a self-calibrating bundle adjustment. This paper utilizes both Gauss-Markov and Gauss-Helmert models for fisheye calibration adjustments.
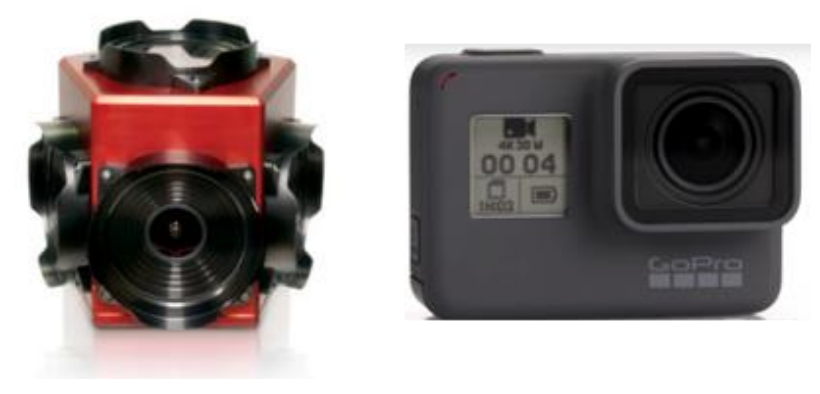

Figure 1. Left: Ladybug5("Ladybug5 360 Degree USB3 Spherical Camera Systems," 2019). Right: GoPro

Hero5(“HERO5 Black Quick Start - Overview,” 2019, p. 5)

\subsection{Literature Review}

Conventional cameras are generally calibrated using the central perspective model. Fisheye lenses do not follow the same model since they have a FOV of $180^{\circ}$ or more, which cannot be described using the central perspective model (Kedzierski and Fryskowska, 2008; Schneider et al., 2009; Luhmann et al., 2017). Fisheye lenses can be modelled using four different projection models: equidistant, equi-solid angle, orthographic, and stereographic projections (Schneider et al., 2009). Equidistant and equi-solid are the most commonly used models and have been found to fit image observations more precisely and provide higher estimated object point coordinate precision when combined with additional distortion parameters (Schneider et al., 2009). Fisheye cameras are typically calibrated in three ways 
(Urban et al., 2015): self-calibrating bundle adjustment, space resection of a single image (Schneider et al., 2009), and 2D checkerboard calibration with multiple images (Kanatani, 2013).

There is some ambiguity in the current research as to what exactly the term wide-angle means and how it relates to fish-eye lens cameras. In Urban (2015) wide-angle lens cameras (such as the GoPro and Ladybug1) are grouped as fish-eye and omnidirectional camera lenses for their calibration methodology. Fraser (1997) recommends the use of additional radial distortion parameters in the central perspective model for wide-angle lens cameras. Brauer-Burchardt and Voss (2001) developed a calibration workflow for dealing with both super-wide angle and fisheye lenses. In Schneider and Forstner (2013), a Ladybug3 multi-camera system was calibrated using points at infinity, and the cameras within the system were treated as non-fisheye (conventional) cameras. Ikeda et al. (2003) modelled the Ladybug camera system as a non-fisheye lens, calibrating it using checkerboards and Tsai's method for corner detection (Tsai, 1987). Some research has modelled wide-angle lens cameras, such as the Ladybug and GoPro, using the central perspective model using additional (more than 3) radial lens parameters to counter-act the extreme distortions, specifically closer to image corners (Balletti et al., 2014). This suggests that there are multiple methods in use for calibrating wide-angle lenses. This ambiguity is understandable considering that wide-angle lens cameras produce images that look similar to full-format fisheye images but can be modelled by the central perspective model.

The fact that multiple models are used by different researchers suggests that there is validity to both models, and both likely provide useful calibration parameters for a variety of applications. However, there are some applications that need the highest degree of accuracy possible. Therefore, if wide-angle lens cameras and camera-systems are going to be utlized for these applications, the most accurate model needs to be determined. Examples of accuracy-dependent applications are robotic manipulation (Nickels et al., 2010; Tamadazte et al., 2011), manufacturing and production part inspection (Jayaweera and Webb, 2010; Schwenke et al., 2002; Shi et al., 2010), and infrastructure deformation monitoring (Detchev et al., 2014; Kwak et al., 2013; Whiteman et al., 2002). This work aims to determine which calibration model is more effective for use in such precision applications.

\section{METHODOLOGY}

Central perspective projection geometry can be expressed via augmented collinearity equations. In the case of wide-angle lenses, radial lens distortions are significantly pronounced. Thus, higher orders of polynomials are needed to model them effectively. Equation 1 describes the augmented collinearity equations commonly used in the literature to model the interior orientation parameters of cameras with wide-angle lenses.

$$
\begin{aligned}
& x_{i j}-c_{x_{m}}+\delta_{x_{i j}}+f_{m} \frac{u_{i j}}{w_{i j}}=0 \\
& y_{i j}-c_{y_{m}}+\delta_{y_{i j}}+f_{m} \frac{v_{i j}}{w_{i j}}=0
\end{aligned}
$$

where

$$
\begin{aligned}
& {\left[\begin{array}{lll}
u_{i j} & v_{i j} & w_{i j}
\end{array}\right]^{T}=\left[\begin{array}{ll}
\boldsymbol{R}_{o}^{j} & -\boldsymbol{R}_{o}^{j} C_{j}
\end{array}\right] \widetilde{\mathrm{X}}_{i}} \\
& m=\text { camera index } \\
& i=\text { tie point index } \\
& j=\text { image index } \\
& o=\text { denotes objects coordinate system }
\end{aligned}
$$

The Gauss-Helmert Model (GHM) is used with these equations because observations and parameters are non-linearly combined. In Equations 1 and $2, \boldsymbol{R}_{o}^{j}\left(\omega_{j}, \varphi_{j}, \kappa_{j}\right)$ is the rotation matrix from the object to the camera coordinate system, $C_{j}$ is the position of the image perspective center in the object coordinate system. Together, they are the exterior orientation parameters (EOPs) of the camera. $\tilde{X}_{i}$ is the Euclidean normalized homogeneous object coordinates of a tie-point and $\left(x_{i j}, y_{i j}\right)$ are the observations of that point in an image. Interior orientation parameters of the cameras include $\left(c_{x_{m}}, c_{y_{m}}, f_{m}\right)$, respectively the principal point offsets and the principal distance, and $\left(\delta_{x_{i j}}, \delta_{y_{i j}}\right)$ are the distortion corrections that are modelled as follows:

$$
\begin{aligned}
\delta_{x_{i j}}= & \left(x_{i j}-c_{x_{m}}\right)\left(k_{1_{m}} r_{i j}^{2}+k_{2_{m}} r_{i j}^{4}+k_{3_{m}} r_{i j}^{6}+k_{4_{m}} r_{i j}^{8}+k_{5_{m}} r_{i j}^{10}\right) \\
& +p_{1_{m}}\left(r_{i j}^{2}+2\left(x_{i j}-c_{x_{m}}\right)^{2}\right)+2 p_{2_{m}}\left(x_{i j}-c_{x_{m}}\right)\left(y_{i j}-c_{y_{m}}\right) \\
& +s_{1_{m}}\left(x_{i j}-c_{x_{m}}\right)+s_{2_{m}}\left(y_{i j}-c_{y_{m}}\right) \\
\delta_{y_{i j}}= & \left(y_{i j}-c_{y_{m}}\right)\left(k_{1_{m}} r_{i j}^{2}+k_{2_{m}} r_{i j}^{4}+k_{3_{m}} r_{i j}^{6}+k_{4_{m}} r_{i j}^{8}+k_{5_{m}} r_{i j}^{10}\right) \\
+ & +p_{2_{m}}\left(r_{i j}^{2}+2\left(y_{i j}-c_{y_{m}}\right)^{2}\right)+2 p_{1_{m}}\left(x_{i j}-c_{x_{m}}\right)\left(y_{i j}-c_{y_{m}}\right)
\end{aligned}
$$

where

$$
\begin{aligned}
& r_{i j}=\sqrt{\left(x_{i j}-c_{x_{m}}\right)^{2}+\left(y_{i j}-c_{y_{m}}\right)^{2}} \\
& k_{1_{m}}, k_{2_{m}}, k_{3_{m}}, k_{4_{m}}, k_{5_{m}}=\text { radial lens distortion } \\
& \text { parameters } \\
& p_{1_{m}}, p_{2_{m}}=\text { decentring lens distortion } \\
& \text { parameters } \\
& s_{1_{m}}, s_{2_{m}}=\text { sensor affine distortion parameters }
\end{aligned}
$$

Fisheye lenses are designed to capture a large field of view, usually over $100^{\circ}$. Thus, it is geometrically impossible to project such a large field on the limited image plane by central perspective projection. Thus, optical fisheye construction cannot be described naively as a deviation from the pinhole model with higher-order polynomials. Therefore, a different geometric model should be used to intrinsically calibrate fisheye cameras precisely enough to make photogrammetric measurements. In fisheye cameras, a projection ray's incidence angle, $\theta$, is different from its ref angle, $\phi$ (Figure 2).

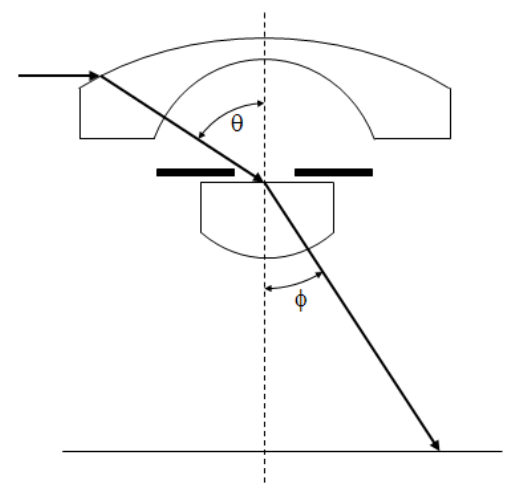

Figure 2. Example of difference in incident $(\theta)$ and resulting $(\phi)$ ray in fisheye lens geometry.

The relation between these two angles defines the type of fisheye projection among four categories of equidistant projection, equisolid-angle projection, orthographic projection, and stereographic projection. Studies have shown that when considering the intrinsic calibration of the sensor-lens combination (additional lens and sensor distortion parameters), the differences between the four fisheye models are negligible (Schneider et al., 2009). Equations 5 and 6 describe the equidistant projection model, which is used in this study to calibrate fisheye cameras. First, the object coordinates are 
projected to the camera coordinate system to measure the projection ray's incidence angle. Then, the geometric relation between the incidence angle and image radial distance is used to establish the mathematical equation between the object and the image space. Additional parameters including parameters of radial lens distortion, decentring lens distortion and sensor affine distortions are also considered.

$$
\begin{aligned}
& x_{i j}-c_{x_{m}}+\delta_{x_{i j}}+f_{m} \frac{u_{i j} \tan ^{-1}\left(\frac{\sqrt{u_{i j}{ }^{2}+v_{i j}{ }^{2}}}{w_{i j}}\right)}{\sqrt{u_{i j}{ }^{2}+v_{i j}{ }^{2}}}=0 \\
& y_{i j}-c_{y_{m}}+\delta_{y_{i j}}+f_{m} \frac{v_{i j} \tan ^{-1}\left(\frac{\sqrt{u_{i j}^{2}+v_{i j}{ }^{2}}}{w_{i j}}\right)}{\sqrt{u_{i j}{ }^{2}+v_{i j}{ }^{2}}}=0
\end{aligned}
$$

To perform self-calibrating bundle adjustment using Equations 5 and 6 , the GHM should be used since image observations $\left(x_{i j}, y_{i j}\right)$ and unknown calibration parameters are nonlinearly combined as shown in Equations 3 and 4. This necessitates additional matrix calculations and operations since the derivatives of the equations should be taken with respect to the image observations as well. Therefore, the fisheye model can alternatively be represented as Equations 7 and 8 (Kannala and Brandt, 2006). Using Equations 7 and 8, the self-calibration bundle adjustment can be formulated via the Gauss Markov Model (GMM). In this study, the calibration precision achievable by these models (Equations 5 and 6 versus Equations 7 and 8 ) as well using the central perspective model will be investigated. In Equations 7 and 8 , parameters

$\left(\beta_{1_{m}}, \beta_{2_{m}}, \beta_{3_{m}}, \beta_{4_{m}}, c_{x_{m}}, c_{y_{m}}, f_{x_{m}}, f_{y_{m}}\right) \quad$ are the intrinsic calibration parameters that need to be determined through the calibration process.

$$
\begin{gathered}
x_{i j}-c_{x_{m}}+f_{x_{m}}\left(\frac{u_{i j} \rho_{i j}}{\sqrt{u_{i j^{2}+v_{i j}{ }^{2}}}}\right)+\beta_{4_{m}}\left(\frac{v_{i j} \rho_{i j}}{\sqrt{u_{i j^{2}+v_{i j}{ }^{2}}}}\right)=0 \\
y_{i j}-c_{y_{m}}+f_{y_{m}}\left(\frac{v_{i j} \rho_{i j}}{\sqrt{u_{i j^{2}+v_{i j} j^{2}}}}\right)=0
\end{gathered}
$$

where

$$
\begin{aligned}
& \rho_{i j}=\alpha_{i j}+\beta_{1_{m}} \alpha_{i j}{ }^{2}+\beta_{2_{m}} \alpha_{i j}{ }^{3}+\beta_{3_{m}} \alpha_{i j}{ }^{4} \\
& \alpha_{i j}=\tan ^{-1}\left(\frac{\sqrt{u_{i j}^{2}+v_{i j}{ }^{2}}}{w_{i j}}\right)
\end{aligned}
$$

Free-network self-calibrating bundle adjustment was performed using inner constraints imposed on object coordinates of ties points and EOPs of the cameras. Since the self-calibration can involve a large number of image observations, specifically in the case of Ladybug system with six cameras, the degree of freedom of the adjustment can be very large. Thus, sparse bundle adjustment should be used to facilitate calculations and data storage. Readers are referred to Shahbazi et al., (2017) for the details of implementing sparse bundle adjustment with GMM or GHM.

\section{EXPERIMENTS}

The imagery was captured from multiple sensors to evaluate the calibration precision of the different methods described in Section 2. The two sensors studies in this paper are the Ladybug 5 (FLIR Systems, Oregon, USA) and the GoPro Hero5. The Ladybug 5 is an omnidirectional multi-camera system composed of 6 wide-angle lens cameras. Each of the Ladybug5's six cameras has a nominal principal distance of $4.4 \mathrm{~mm}$, a sensor size of $2448 \times 2048$, and a pixel pitch of $0.00345 \mathrm{~mm}$, giving it an approximate FOV of $103^{\circ}$. The GoPro Hero5 is a very-wideangle lens camera with a $3 \mathrm{~mm}$ principal distance, a sensor size of $4000 \times 3000$ pixels, and a pixel pitch of $0.0015 \mathrm{~mm}$, giving it an approximate FOV of $103^{\circ}$.

Imagery from both camera systems was captured in a calibration room having controlled lighting and temperature. This room's (dimensions $11 \mathrm{~m} \mathrm{x} 11 \mathrm{~m} \mathrm{x} 4 \mathrm{~m}$ ) walls, ceiling, and floor are covered in un-coded circular photogrammetry targets of which 232 are $125 \mathrm{~mm}$ radius targets made from $4 \mathrm{~mm}$ thick BubbleX plastic, and 59 are paper targets of $40 \mathrm{~mm}$ radius. Smaller targets also exist in the calibration room, but they were ignored in these experiments.

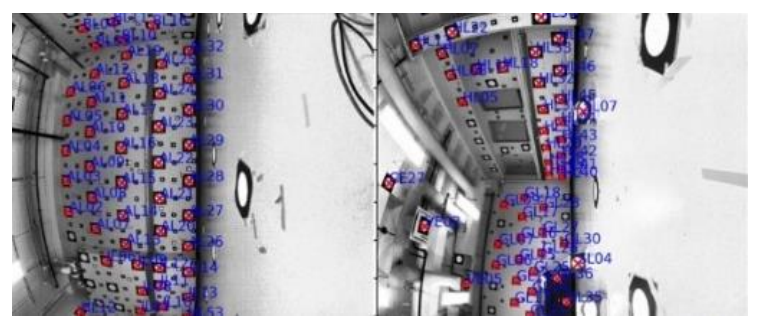

Figure 3. Example of Ladybug5 imagery with measured targets.

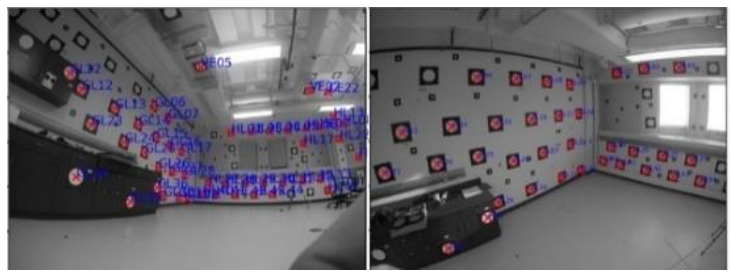

Figure 4. Example of GoPro Hero5 imagery with measured targets.

Automated target measurement and recognition were used to generate observations for use in the bundle adjustments, using a method based on previous work done by the author. Measured recognized targets can be seen in Figures 3 and 4. The targets in the calibration space were imaged with a Faro Focus 3D laser scanner, and the centre coordinates of each target were extracted by fitting a circle to the edge points, as seen in Figure 5 (Lichti et al., In Press). The average precision of this target fitting method on the surveyed targets is $1.4 \mathrm{~mm} \pm 0.4 \mathrm{~mm}$ at $2 \sigma$. From the Ladybug5 system, 261 images were captured from the six cameras. Images were taken from different heights and with orthogonal roll angles utilizing convergent geometry. A total of 118 targets were accurately measured on at least 15 images, resulting in some 12,250 observation equations. In addition, an independent set of 61 images were captured, with 213 targets observed in at least 3 images for validation purposes. On these check images, a separate bundle adjustment was performed where the 3D object-space coordinates of four targets measured via a laser scanner were considered as additional observations and the interior orientation parameters estimated through the selfcalibrating free-network bundle adjustments were considered known. As a result of this check adjustment, the 3D object-space coordinates of the other targets were estimated. The difference of the estimated coordinates for 187 checkpoints from their reference coordinates observed using a laser scanner was calculated. In the following paragraphs, these differences are 
referred to as the object-space coordinate differences on checkpoints.
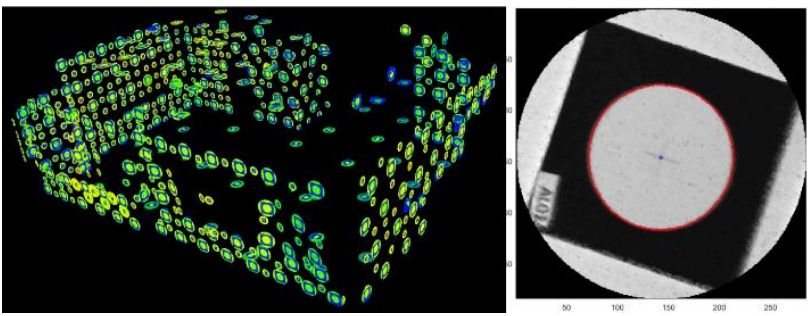

Figure 5. a) Left: Display of object space coordinate survey using laser scan data b) Right: close up of laser scanned targets centre being determined in the laser scan data.

The GoPro Hero5 was used on very wide mode to capture 44 images, from a variety of angles and heights, utilizing convergent geometry, and using a smaller section of the calibration space. A total of 67 targets observed in at least 6 images were accurately measured on these images, resulting in some 2,200 observation equations. Fewer targets were used because the GoPro is not an omnidirectional camera system, and thus can be calibrated using only a few walls of the calibration space. In addition to images taken using the 3D target-field, images were also taken using a checkerboard. Nineteen images were taken of a checkerboard target, with the checkboard covering most of the GoPro's FOV, convergent imagery, and rotations about the optical axis of the camera. A sample of the images used in the calibration can be seen in Figure 6. A total of 132 corners exist on the checkerboard pattern that were observed in at least 14 images resulting in 2,347 observation equations. In addition, an independent set of 18 images were captured from the 3D target-field, over which 59 targets were observed in at least 3 images for validation purposes.

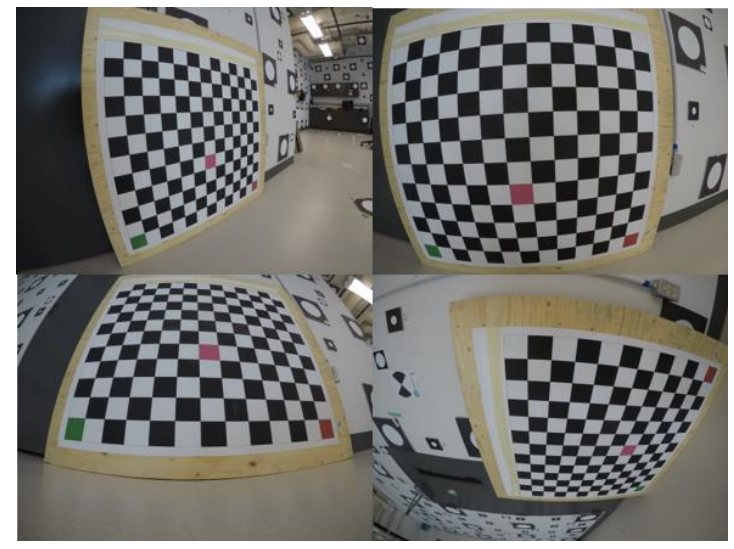

Figure 6. Sample of checkboard target calibration imagery.

\section{RESULTS}

\subsection{Calibration of Ladybug5 using different models}

Bundle adjustments were run using observations measured in the Ladybug5 images and utilizing the three different models described in Section 2. The three models are the central perspective augmented collinearity model, the fisheye GHM, and the fisheye GMM. The central perspective model was tested using three variations, each having a different number of radial lens distortion parameters. The first variation uses the standard three radial lens distortion parameters, the second uses four, and the last variation uses five. The mean and root mean square
(RMS) of the image observations were used to evaluate the precision of the adjustment.

Tables 1 and 2 demonstrate that the GHM fisheye model provides smaller image space residuals, both in the precision of the selfcalibrating bundle adjustment and in the check image adjustment. It is notable that the Fisheye GHM adjustment provides the smaller residuals while having the same number of camera model parameters as the standard K1-3 collinearity model. This suggests the Fisheye GHM models the camera more effectively. Both fisheye models generate smaller residuals than any of the central perspective models. Amongst the central perspective models, the model with five radial lens distortion parameters provides the best fit of residuals. This is likely caused by the heavy distortion near the edges of the images, which requires more than the standard parameters to compensate for.

Table 1. Calibration precision of Ladybug5 camera system using various calibration models in terms of image residuals.

\begin{tabular}{|c|c|c|c|c|c|}
\hline Model & $\begin{array}{c}\text { K1- } \\
5\end{array}$ & K1-4 & $\begin{array}{c}\text { K1- } \\
3\end{array}$ & $\begin{array}{c}\text { Fisheye } \\
\text { (GHM) }\end{array}$ & $\begin{array}{c}\text { Fisheye } \\
(\text { GMM) }\end{array}$ \\
\hline $\begin{array}{c}\text { \# of IOPs in } \\
\text { Camera } \\
\text { Model }\end{array}$ & 12 & 11 & 10 & $\mathbf{1 0}$ & 8 \\
\hline $\begin{array}{c}\text { Mean } \\
\text { magnitude } \\
\text { (pixels) }\end{array}$ & 0.23 & 0.26 & 0.61 & $\mathbf{0 . 1 2}$ & 0.19 \\
\hline $\begin{array}{c}\text { RMS } \\
\text { (pixels) }\end{array}$ & 0.34 & 0.45 & 1.40 & $\mathbf{0 . 1 5}$ & 0.23 \\
\hline
\end{tabular}

Table 2. Precision on check images of ladybug camera-system in controlled adjustment using the calibration parameters estimated via various calibration models.

\begin{tabular}{|c|c|c|c|}
\hline Ladybug (check) & K1-5 & $\begin{array}{c}\text { Fisheye } \\
\text { (GHM) }\end{array}$ & $\begin{array}{c}\text { Fisheye } \\
\text { (GMM) }\end{array}$ \\
\hline Mean magnitude (pixels) & 0.22 & $\mathbf{0 . 1 3}$ & 0.20 \\
\hline RMS (pixels) & 0.36 & $\mathbf{0 . 1 5}$ & 0.24 \\
\hline
\end{tabular}

Table 3. RMS of object-space coordinate differences of checkpoints for the Ladybug5 camera in check adjustment using parameters estimated from various models.

\begin{tabular}{|c|c|c|}
\hline Model & Axis & RMS (mm) \\
\hline \multirow{4}{*}{ K1-5 } & X & 3.9 \\
\cline { 2 - 3 } & Y & 4.2 \\
\cline { 2 - 3 } & Z & 2.5 \\
\hline \multirow{2}{*}{ Fisheye (GHM) } & X & $\mathbf{3 . 4}$ \\
\cline { 2 - 3 } & Y & $\mathbf{3 . 3}$ \\
\cline { 2 - 3 } & $\mathbf{Z}$ & $\mathbf{2 . 4}$ \\
\hline Fisheye (GMM) & $\mathrm{X}$ & 6.1 \\
\cline { 2 - 3 } & $\mathrm{Y}$ & 5.3 \\
\cline { 2 - 3 } & $\mathrm{Z}$ & 3.8 \\
\hline
\end{tabular}

Table 3 shows that the fisheye GHM generates smaller object space coordinate differences in check images, however the small magnitude of this difference it is not statistically significant due to the surveyed accuracy of the targets. It does indicate that both the central perspective model and the GHM have comparable object space accuracy, but GHM has superior image space residuals. This seems to indicate that the cameras within the Ladybug camera system can be modelled more accurately using a fisheye model than a central perspective model even when five lens distortion parameters are considered. It is interesting to note that the GMM, which is used in by both OpenCV ("OpenCV library," 2019) and Pix4D software ("Professional photogrammetry and drone mapping software," 2019), has 
significantly worse object-space coordinate differences than both other models. This suggests that for precision applications this model may not be ideal. The Fisheye GHM outperforms the central perspective model in terms of image observation residuals and outperforms the GMM in terms of object space check coordinate accuracy. In the case of the Ladybug5 system, the GHM gives the best performance of any model.

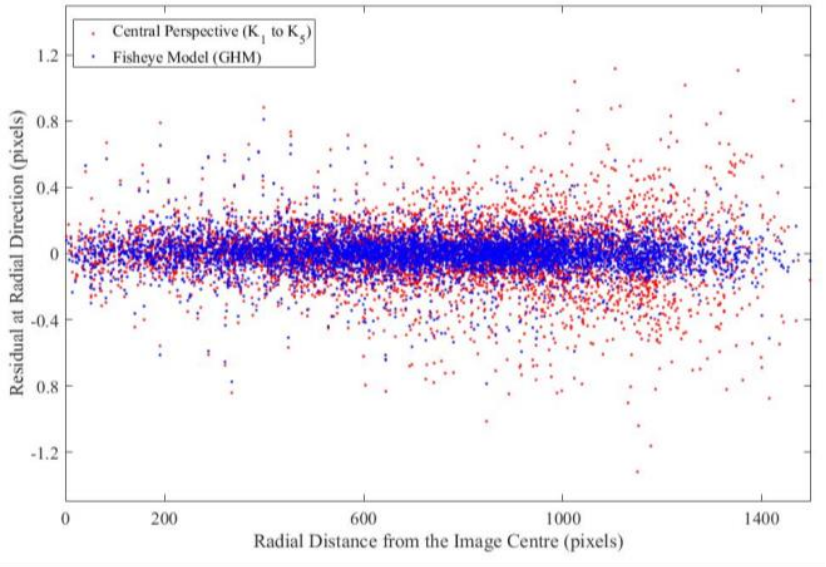

Figure 7. Ladybug image residual absolute magnitudes in the radial direction between the central perspective model and fisheye GHM.
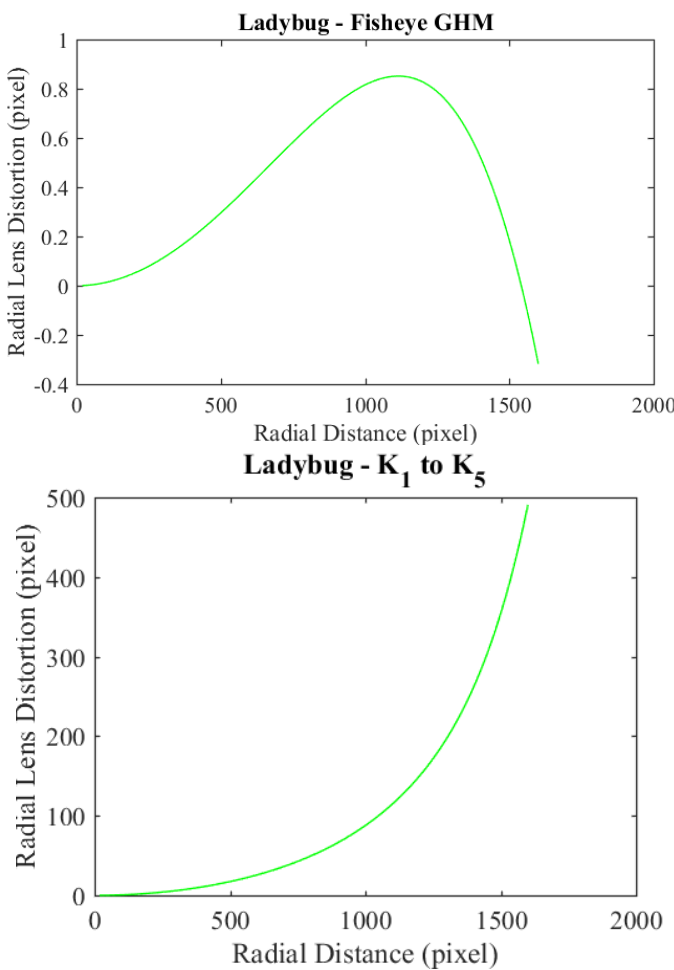

$\mathrm{b}$

Figure 8. Radial lens distortions of Ladybug5 using a) Fisheye GHM and b) Central Perspective K1-K5.

Figure 7 shows the radial component of the residuals from the central perspective model which has a greater spread at larger radial distances (greater than 800 pixels) than the GHM. In contrast, the fisheye model residuals are distributed independently of the radial distance. This demonstrates the effectiveness of the fisheye GHM for modelling the Ladybug's cameras. The lenses of the Ladybug5 camera-system are usually described as wide-angle lenses and modelled using the central perspective model. However, they appear to be modelled more appropriately as fisheye lenses. The central perspective model with additional radial distortion parameters can certainly model the Ladybug, but for metrology or precision-mapping applications, the fisheye GHM provides superior residual fit. A similar trend between the fisheye GHM and central perspective model can be seen in their radial distortion profiles (Figure 8). In the fisheye model, the radial lens distortions are much smaller in magnitude than in the central perspective model. This is caused by the fisheye model compensating for a large portion of the distortions through the equidistant geometric projection while they are considered as excessive radial lens distortions by the central perspective model.

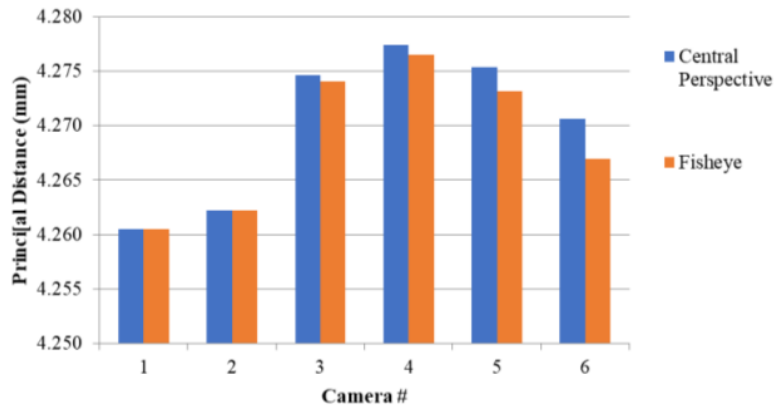

Figure 9. Principal distances of Cameras in Ladybug5 multicamera system determined by different adjustment models

Each camera in the Ladybug system has its interior orientation parameters (IOPs) determined, based on the central perspective model and the GHM. It is worth noting that the fisheye model models the principal distances as being shorter than the central perspective model in 4 of the 6 cameras (Figure 9).

\subsection{Calibration of GoPro Hero5 using different models}

Bundle adjustments were run using observations measured in the GoPro Hero5 images and utilizing the same 5 models as on the Ladybug5. As with the Ladybug5 dataset, the fisheye GHM provides the best fit for the image observations, as can be seen in Table 4.

Table 4.Calibration Precision of GoPro Hero5 camera system using various calibration models in terms of image residuals, based on 3D target field observations.

\begin{tabular}{|c|c|c|c|c|c|}
\hline Model & $\begin{array}{c}\text { K1- } \\
5\end{array}$ & $\begin{array}{c}\text { K1- } \\
4\end{array}$ & $\begin{array}{c}\text { K1- } \\
3\end{array}$ & $\begin{array}{c}\text { Fisheye } \\
(\text { GHM })\end{array}$ & $\begin{array}{c}\text { Fisheye } \\
(\text { GMM })\end{array}$ \\
\hline $\begin{array}{c}\text { \# of IOPs in } \\
\text { Camera Model }\end{array}$ & 12 & 11 & 10 & $\mathbf{1 0}$ & 8 \\
\hline $\begin{array}{c}\text { Mean magnitude } \\
\text { (pixels) }\end{array}$ & 0.31 & 0.32 & 0.48 & $\mathbf{0 . 2 1}$ & 0.24 \\
\hline RMS(pixels) & 0.44 & 0.45 & 0.76 & $\mathbf{0 . 2 7}$ & 0.30 \\
\hline
\end{tabular}

The difference between the GHM and GMM is less pronounced in the GoPro dataset than in the Ladybug dataset as well, which could be due to there being fewer images in the GoPro dataset, or due to the different configurations of the images. In the GoPro dataset, the central perspective model has a better fit than it did in the Ladybug dataset. The results on the check images from the check adjustment (Table 5) show that the fisheye GHM has the smallest image space residuals compared to other adjustment models. In terms of image space residuals, fisheye GHM outperforms both other models and outperforms central perspective models by a significant margin. While the fisheye 
GHM clearly has the best model fit in terms of image residuals, the results are less definitive in the object space. Table 6 shows the results of the object space coordinate differences in check adjustments using parameters from different adjustment models for the GoPro. Again, the accuracy of the target surveying method means that the differences between the models in terms of object space coordinates differences are not statistically significant. Overall, the results of the GoPro are not as clear as the Ladybug, in terms of which model provides the best results. The fisheye GHM has the smallest total residual size, but not by a large margin over either of the other adjustments. It may be the case that the GoPro Hero5 is a camera that can be effectively modelled by either the central perspective model or a Fisheye model. The differences in the total residual RMS between the different models are on the order of $0.01 \mathrm{~mm}$. These differences are too small to determine if there are any significant differences between the models since the accuracy of the surveying method to collect the ground-truth data about checkpoints is not in the order of $1 / 100$ of a millimetre. It is also a possibility that the configuration of imagery used for the check images was not strong enough to discern differences between the methods.

Table 5. Precision on check images of GoPro camera in check adjustment using the calibration parameters estimated via various calibration models, based on 3D target-field observations.

\begin{tabular}{|c|c|c|c|}
\hline GoPro (check) & K1-5 & $\begin{array}{c}\text { Fisheye } \\
(\mathrm{GHM})\end{array}$ & $\begin{array}{c}\text { Fisheye } \\
(\mathrm{GMM})\end{array}$ \\
\hline Mean magnitude (pixels) & 0.83 & $\mathbf{0 . 5 7}$ & 0.60 \\
\hline RMS (pixels) & 1.11 & $\mathbf{0 . 7 0}$ & 0.74 \\
\hline
\end{tabular}

Table 6. RMS of object-space residuals on checkpoints for GoPro camera in check adjustment using parameters estimated from various models, based on 3D target field observations.

\begin{tabular}{|l|c|c|}
\hline Model & Axis & $\begin{array}{c}\text { RMS } \\
(\mathrm{mm})\end{array}$ \\
\hline K1-5 & $\mathrm{X}$ & 3.2 \\
\cline { 2 - 3 } & $\mathrm{Y}$ & 3.2 \\
\cline { 2 - 3 } & $\mathrm{Z}$ & 2.3 \\
\cline { 2 - 3 } & Total & 5.1 \\
\hline \multirow{4}{*}{$\begin{array}{l}\text { Fisheye } \\
\text { (GHM) }\end{array}$} & $\mathrm{X}$ & 3.0 \\
\cline { 2 - 3 } & $\mathrm{Y}$ & 3.3 \\
\cline { 2 - 3 } & $\mathrm{Z}$ & 2.3 \\
\cline { 2 - 3 } & Total & $\mathbf{5 . 0}$ \\
\hline \multirow{3}{*}{$\begin{array}{l}\text { Fisheye } \\
\text { (GMM) }\end{array}$} & $\mathrm{X}$ & 3.3 \\
\cline { 2 - 3 } & $\mathrm{Y}$ & 3.1 \\
\cline { 2 - 3 } & $\mathrm{Z}$ & 2.4 \\
\cline { 2 - 3 } & Total & 5.1 \\
\hline
\end{tabular}

Similar to the results of the Ladybug dataset, the spread of the of the GoPro's residuals along the radial direction increase with the distance from the image centre for the central perspective model (Figure 10). The effect is not as drastic as in the Ladybug data, but there is a definite trend. The fisheye GHM has both lower residuals and no trend with regards to the radial distance. Unlike the Ladybug5 results, the GMM model seems more comparable in the object point coordinate differences to the other two models. Figure 11 shows that the GHM compensates for a significant amount of the radial lens distortion, although the amount remaining to be corrected by the distortion parameters is much higher than in the case of the Ladybug (less than 2 pixels for the Ladybug, and up to 180 pixels for the GoPro).

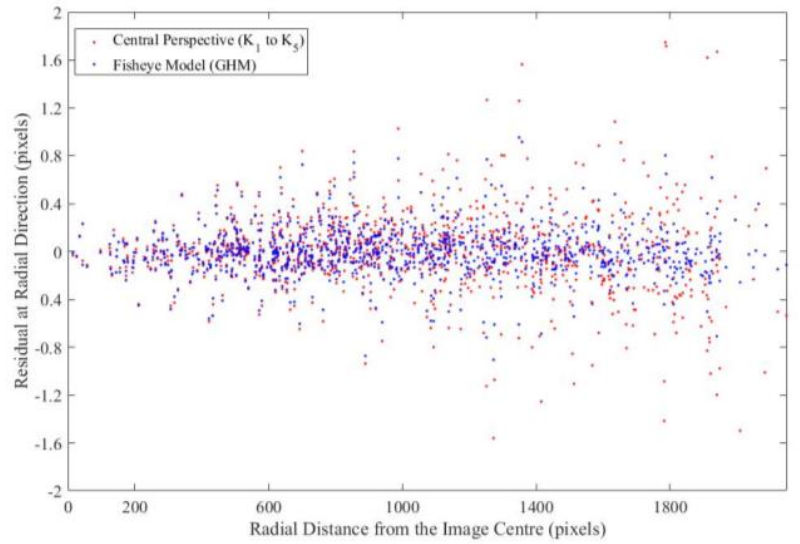

Figure 10. GoPro image residual absolute magnitudes in the radial direction between the central perspective model and fisheye GHM.

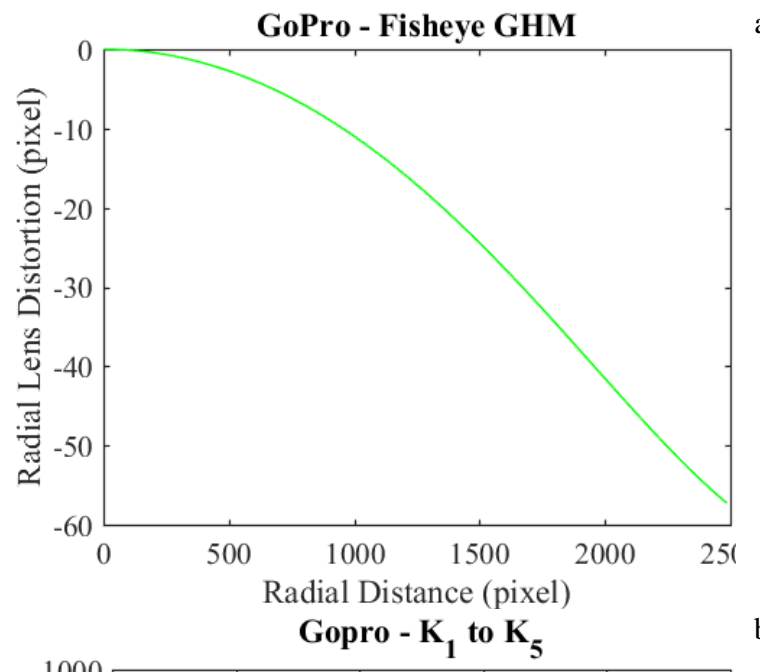
a

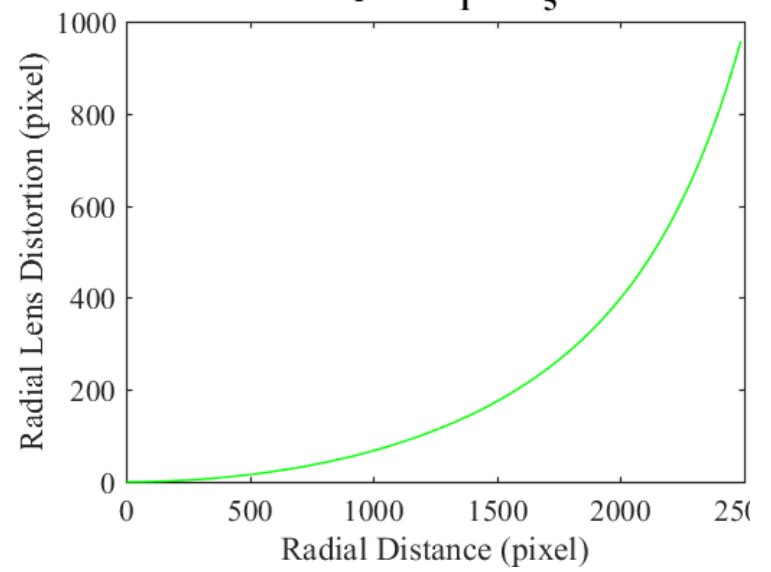

Figure 11. Radial lens distortions of GoPro using a) Fisheye GHM and b) Central Perspective K1-K5.

For the GoPro camera, self-calibrating bundle adjustments with different models were also performed using observations derived from a checkboard target pattern. Due to the checkerboard pattern being much smaller in dimension, the images taken of it had larger intersection angles, giving it more convergent geometry than that taken in the 3D target field. The 3D target field was initially designed for use with the Ladybug5 system, which is an omnidirectional camera system. Thus, it was important to have a large range of coverage around the camera system. This led to targets being spread throughout the room, rather than 
concentrated in one area. It seems likely that the denser spread of targets and less stable network geometry are the reasons behind the checkerboard calibrations having slightly smaller imageobservation residuals compared to the 3D target-field (Table 7). The relative quality of the different adjustment models remains the same as the calibrations using 3D target-field observations however, with the fisheye GHM out-performing the other models. Table 8 shows that the object-space coordinate differences on checkpoints are different from their counterparts in Table 6. For the checkerboard observation adjustments, the RMS of the checkpoints is lower than those from the 3D targetfield adjustments. In the case of the checkerboard, the central perspective model has the lowest total residuals, by a slight margin. This margin is very small in comparison to the accuracy of the surveying method used, making it difficult to make definitive statements about the differences in object point coordinate differences between the two models. However, the fisheye GHM had the best image observation residual fit in all experiments. The check image precision for the checkerboard imagery was almost identical in values to those of the 3D target field calibration.

Table 7. Calibration Precision of GoPro Hero5 camera system using various calibration models in terms of image residuals, based on checkerboard pattern observations.

\begin{tabular}{|c|c|c|c|c|c|}
\hline GoPro & $\begin{array}{c}\text { K1- } \\
5\end{array}$ & $\begin{array}{c}\text { K1- } \\
4\end{array}$ & $\begin{array}{c}\text { K1- } \\
3\end{array}$ & $\begin{array}{c}\text { Fisheye } \\
\text { (GHM) }\end{array}$ & $\begin{array}{c}\text { Fisheye } \\
(\text { GMM })\end{array}$ \\
\hline $\begin{array}{c}\text { \# of IOPs in } \\
\text { Camera Model }\end{array}$ & 12 & 11 & 10 & $\mathbf{1 0}$ & 8 \\
\hline $\begin{array}{c}\text { Mean magnitude } \\
\text { (pixels) }\end{array}$ & 0.18 & 0.19 & 0.23 & $\mathbf{0 . 1 4}$ & 0.15 \\
\hline RMS(pixels) & 0.22 & 0.23 & 0.33 & $\mathbf{0 . 1 6}$ & 0.18 \\
\hline
\end{tabular}

Table 8. RMS of object-space residuals on checkpoints for GoPro camera in check adjustment using parameters estimated from various models, based on checkerboard pattern observations.

\begin{tabular}{|c|c|c|}
\hline Model & Axis & $\begin{array}{c}\text { RMS } \\
(\mathrm{mm})\end{array}$ \\
\hline \multirow{3}{*}{ K1-5 } & $\mathrm{X}$ & 2.8 \\
\cline { 2 - 3 } & $\mathrm{Y}$ & 3.1 \\
\cline { 2 - 3 } & $\mathrm{Z}$ & 2.2 \\
\cline { 2 - 3 } & Total & $\mathbf{4 . 7}$ \\
\hline \multirow{3}{*}{$\begin{array}{c}\text { Fisheye } \\
\text { (GHM) }\end{array}$} & $\mathrm{X}$ & 2.4 \\
\cline { 2 - 3 } & $\mathrm{Y}$ & 3.5 \\
\cline { 2 - 3 } & $\mathrm{Z}$ & 2.3 \\
\cline { 2 - 3 } & Total & 4.8 \\
\hline \multirow{3}{*}{$\begin{array}{c}\text { Fisheye } \\
\text { (GMM) }\end{array}$} & $\mathrm{X}$ & 3.6 \\
\cline { 2 - 3 } & $\mathrm{Y}$ & 3.1 \\
\cline { 2 - 3 } & $\mathrm{Z}$ & 2.5 \\
\cline { 2 - 3 } & Total & 5.4 \\
\hline
\end{tabular}

\section{CONCLUSION}

This work studied the effects of modelling wide-angle lenses using both the conventional central perspective model and fisheye models, to determine which models the distortions more accurately. A series of images were captured using a Ladybug5 and a GoPro Hero5. Multiple free-network, self-calibrating bundle adjustments were performed using five different models on both cameras. The most significant comparison was between the central perspective adjustment with five radial distortion parameters and the GHM fisheye adjustment. It was found that the central perspective model had a significant uncompensated radial component in the image observation residuals that was not present in the fisheye GHM. The fisheye GHM significantly reduced image observation residuals compared to the central perspective model. The Fisheye GHM calibration achieved superior results to the collinearity model and utilized fewer interior orientation parameters to do so. For the Ladybug5, the RMS of image residuals using the GHM was 0.15 pixels compared to 0.34 pixels for the central perspective model. For the GoPro, the RMS using the fisheye GHM was 0.27 pixels compared to 0.44 pixels for the central perspective model. In the Ladybug multi-camera system, the fisheye GHM and central perspective models produce similar object-space checkpoint coordinate differences in a check adjustment and outperform the GMM significantly. These results were not confirmed for the GoPro Hero5, in which all models had similar object-space residuals. The GoPro may be a camera that straddles the line between the central perspective model and the fisheye model and can thus be accurately modelled by either, whereas the Ladybug is most effectively modelled using the GHM.

\section{REFERENCES}

Balletti, C., Guerra, F., Tsioukas, V., Vernier, P., 2014. Calibration of Action Cameras for Photogrammetric Purposes. Sensors ,14, 17471-17490. doi.org/10.3390/s140917471.

Boulianne, M., Nolette, C., Agnard, J.-P., Brindamour, M., 1997. Hemispherical Photographs Used for Mapping Confined Spaces. Photogrammetric Engineering \& Remote Sensing,63, 11031109.

Brauer-Burchardt, C., Voss, K., 2001. A new algorithm to correct fish-eye- and strong wide-angle-lens-distortion from single images. Presented at the Proceedings 2001 International Conference on Image Processing (Cat. No.01CH37205), 1, 225-228. doi.org/10.1109/ICIP.2001.958994.

Cui, T., Ji, S., Shan, J., Gong, J., Liu, K., 2016. Line-Based Registration of Panoramic Images and LiDAR Point Clouds for Mobile Mapping. Sensors,17. doi.org/10.3390/s17010070.

da Silva, S.P.P., Marinho, L.B., Almeida, J.S., Rebouças Filho, P.P., 2017. A Novel Approach for Mobile Robot Localization in Topological Maps Using Classification with Reject Option from Structural Co-occurrence Matrix, in: Felsberg, M., Heyden, A., Krüger, N. (Eds.), Computer Analysis of Images and Patterns, Lecture Notes in Computer Science. Springer International Publishing, 3-15.

Detchev, I., Habib, A., He, F., El-Badry, M., 2014. Deformation monitoring with off-the-shelf digital cameras for civil engineering fatigue testing. ISPRS - International Archives of the Photogrammetry, Remote Sensing and Spatial Information Sciences, XL-5, 195-202. doi.org/10.5194/isprsarchives-XL-5195-2014.

Fraser, C.S., 1997. Digital camera self-calibration. ISPRS Journal of Photogrammetry and Remote Sensing,52, 149-159. doi.org/10.1016/S0924-2716(97)00005-1.

HERO5 Black Quick Start - Overview, 2019. URL https://gopro.com/channel/product-stories/hero5-black-quickstart-overview (accessed 3.19.19). 
Ikeda, S., Sato, T., Yokoya, N., 2003. High-resolution panoramic movie generation from video streams acquired by an omnidirectional multi-camera system, in: Proceedings of IEEE International Conference on Multisensor Fusion and Integration for Intelligent Systems, MFI2003. 155-160. doi.org/10.1109/MF I-2003.2003.1232649.

Jayaweera, N., Webb, P., 2010. Metrology-assisted robotic processing of aerospace applications. International Journal of Computer Integrated Manufacturing, 23, 283-296. doi.org/10.1080/09511920903529255.

Kanatani, K., 2013. Calibration of Ultrawide Fisheye Lens Cameras by Eigenvalue Minimization. IEEE Transactions on Pattern Analysis and Machine Intelligence, 35, 813-822. doi.org/10.1109/TPAMI.2012.146.

Kannala, J., Brandt, S.S., 2006. A generic camera model and calibration method for conventional, wide-angle, and fish-eye lenses. IEEE Transactions on Pattern Analysis and Machine Intelligence, 28, 1335-1340. doi.org/10.1109/TPAMI.2006.153.

Kedzierski, M., Fryskowska, A., 2008. Precise Method of Fisheye Lens Calibration. The International Archives of the Photogrammetry, Remote Sensing and Spatial Information Sciences,XXXVII. Part B5.

Kwak, E., Detchev, I., Habib, A., El-Badry, M., Hughes, C., 2013. Precise Photogrammetric Reconstruction Using ModelBased Image Fitting for 3D Beam Deformation Monitoring. Journal of Surveying Engineering, 139, 143-155. doi.org/10.1061/ (ASCE)SU.1943-5428.0000105.

Ladybug5 360 Degree USB3 Spherical Camera Systems, 2019. URL https://www.ptgrey.com/ladybug5-360-degree-usb3spherical-camera-systems (accessed 3.19.19).

Lichti, D., Jahraus, A., Hartzell, P., 2019. (In Press) New approach for low-cost TLS target measurement. ASCE Journal of Surveying Engineering.

Luhmann, T., Fraser, C., Maas, H.-G., 2016. Sensor modelling and camera calibration for close-range photogrammetry. ISPRS Journal of Photogrammetry and Remote Sensing, Theme issue " State-of-the-art in photogrammetry, remote sensing and spatial information science", 115, 37-46. doi.org/10.1016/j.isprsjprs. 2015.10.006.

Nickels, K., DiCicco, M., Bajracharya, M., Backes, P., 2010. Vision guided manipulation for planetary robotics - position control. Robotics and Autonomous Systems, 58, 121-129. doi.org/10.1016/j.robot.2009.07.029.

OpenCV library, 2019. URL https://opencv.org/ (accessed 4.7.19).

Possoch, M., Bieker, S., Hoffmeister, D., Bolten, A., Schellberg, J., Bareth, G., 2016. Multi-Temporal Crop Surface Models Combined With The Rgb Vegetation Index From Uav-Based Images For Forage Monitoring In Grassland. ISPRS International Archives of the Photogrammetry, Remote Sensing and Spatial Information Sciences, XLI-B1, 991-998. doi.org/10.5194/isprsarchives-XLI-B1-991-2016.

Professional photogrammetry and drone mapping software, 2019. . Pix4D. URL https://www.pix4d.com/ (accessed 4.7.19).
Puente, I., González-Jorge, H., Arias, P., Armesto, J., 2012. Land-Based Mobile Laser Scanning Systems: A Review, in: ISPRS - International Archives of the Photogrammetry, Remote Sensing and Spatial Information Sciences,163-168. doi.org /10.5194/isprsarchives-XXXVIII-5-W12-163-2011.

Schneider, D., Schwalbe, E., Maas, H.-G., 2009. Validation of geometric models for fisheye lenses. ISPRS Journal of Photogrammetry and Remote Sensing, 64, 259-266. doi.org/10. 1016/j.isprsjprs.2009.01.001.

Schneider, J., Forstner, W., 2013. Bundle Adjustment and System Calibration with Points at Infinity for Omnidirectional Cameras. Institute of Geodesy and Geoinformation, University of Bonn, Germany.

Schwenke, H., Neuschaefer-Rube, U., Pfeifer, T., Kunzmann, H., 2002. Optical Methods for Dimensional Metrology in Production Engineering. CIRP Annals, 51, 685-699. doi.org/10.1016/S0007 -8506(07)61707-7.

Shahbazi, M., Sohn, G., Théau, J., Ménard, P., 2017. Robust structure-from-motion computation: Application to open-pit mine surveying from unmanned aerial images. J. Unmanned Veh. Sys., 5, 126-145. doi.org/10.1139/juvs-2016-0027.

Shi, Q., Xi, N., Zhang, C., 2010. Develop a Robot-Aided Area Sensing System for 3D Shape Inspection. J. Manuf. Sci. Eng, 132, 014502-014502-4. doi.org/10.1115/1.4000756.

Tamadazte, B., Piat, N.L., Dembélé, S., 2011. Robotic Micromanipulation and Microassembly Using Monoview and Multiscale Visual Servoing. IEEE/ASME Transactions on Mechatronics, 16, 277-287. doi.org/10.1109/TMECH.2010.204 900.

Tsai, R., 1987. A versatile camera calibration technique for highaccuracy 3D machine vision metrology using off-the-shelf TV cameras and lenses. IEEE Journal on Robotics and Automation 3, 323-344. https://doi.org/10.1109/JRA.1987.1087109

Urban, S., Leitloff, J., Hinz, S., 2015. Improved wide-angle, fisheye and omnidirectional camera calibration. ISPRS Journal of Photogrammetry and Remote Sensing, 108, 72-79. doi.org/10 .1016/j.isprsjprs.2015.06.005

Whiteman, T., Lichti, D.D., Chandler, I., 2002. Measurement Of Deflections In Concrete Beams By Close-Range Digital Photogrammetry. Presented at the Symposium of Geospaial Theory, Processing and Applications, Ottawa, Canada, p. 9. 